\title{
5 Automotive Health - Was das Automobil mit Gesundheit zu tun hat
}

\author{
David Matusiewicz und Manfred Knye
}

\section{Automotive Health - Eine Einführung in mobile Health}

Automotive Health beschreibt die Verschmelzung zweier klassischer Branchen: der Automobilindustrie und des Gesundheitswesens. Die Elektrifizierung des Autos ändert das Produkt grundsätzlich und nachhaltig, allerdings zunächst einmal nicht das Geschäftsmodell und die Position des Herstellers beim Kunden. Die Digitalisierung hingegen ändert das Produkt und das Geschäftsmodell. Dies schafft neue Konkurrenten, verringert den Entwicklungsvorsprung der etablierten Produzenten und braucht neue Kompetenzen, um im Wettbewerb bestehen zu können.

Wenn das erste „iPhone auf Rädern“ zu kaufen sein wird, wird die Automobilindustrie dann nur noch Zulieferer der Mobilitätsanbieter werden?

Das digitalisierte/selbstfahrende Auto ist dann wahrscheinlich nicht mehr das Endprodukt, sondern vielmehr „nur“ Mittel zum Zweck. Die Mobilität als solches wird aus Kundensicht zum Endprodukt. Dies würde die Position des Automobilherstellers in Zukunft drastisch verändern. Die bisherigen Hersteller könnten dann entweder zum Mobilitätsanbieter avancieren oder sich in der Rolle des Zulieferers für Mobilitätsanbieter wiederfinden. Das Gesundheitswesen befindet sich ebenfalls mitten in der digitalen Transformation. Die etablierten Akteure transformieren ihre Geschäftsmodelle und neue Player bringen innovative und teilweise disruptive Lösungen in den Markt (s. Abb. 1). 


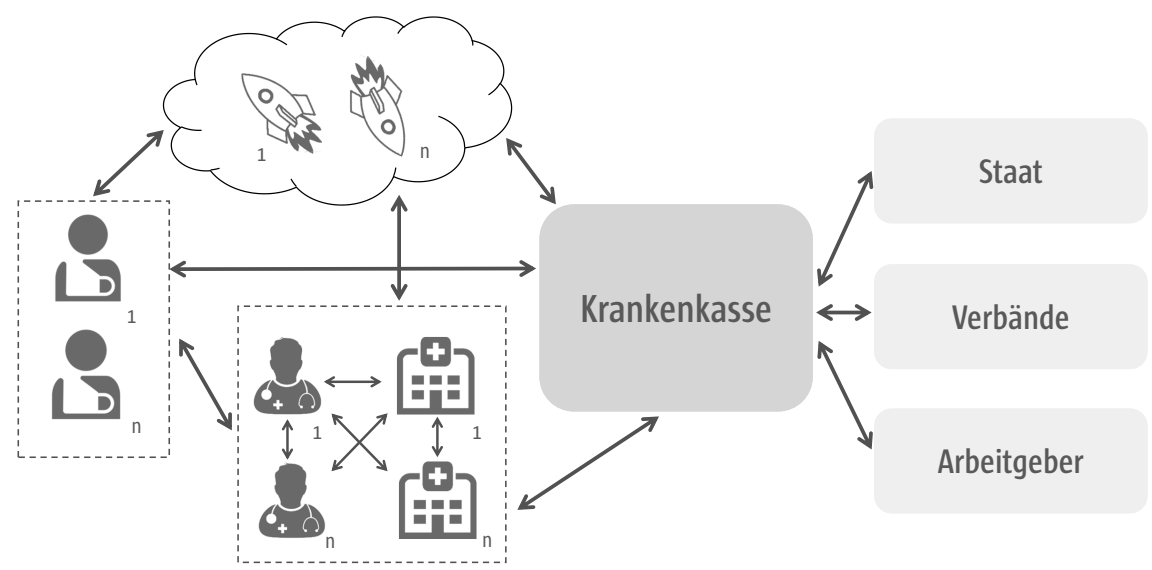

Abb. 1 Neue Player (hier in der Wolke mit den Raketen dargestellt) werden den klassischen Markt aus Patienten, Ärzten, Krankenhäusern, Krankenkassen und weiteren Akteuren aufmischen.

Für alle großen Internetkonzerne scheint das Thema Gesundheit ein wichtiges Zukunftsthema zu sein: Google Health beschäftigt derzeit über 150 Mitarbeiter und versucht beispielsweise mit den Google Lenses Blutzucker über Kontaktlinsen zu messen. Bei Apple ist durchgedrungen, dass der Konzern mit einem Armband für die Apple Watch in $\mathrm{Zu}-$ kunft optisch Blutzucker messen möchte. Boston Dynamics versucht mit Robotern die Pflege der Zukunft sicherzustellen und IBM investiert rund 300 Mio. \$ pro Jahr in Gesundheits-Datenmanagement. Der Supercomputer Watson (IBM) forscht in der Krebsforschung und SAP in der Sequenzierung der Genome von Patienten im Sinne der personalisierten bzw. stratifizierten Medizin. Für Microsoft Health ist der 2. Gesundheitsmarkt hinsichtlich Wearables, Apps und Gesundheitsplattformen interessant. Doch die Gesundheitsbranche gilt bisweilen nicht gerade als großer Innovator, wie das Beispiel der elektronischen Gesundheitskarte (eGK) gezeigt hat. Auch die Tatsache, dass die Gesundheitsbranche einen Innovationsfonds braucht, um innovativ zu sein, ist ein Bekenntnis zur Rückständigkeit. Somit könnte man ein Zwischenfazit ziehen, dass die Gesundheitsbranche sich derzeit zwischen tradiertem Stillstand und disruptiven Sprüngen befindet. Für viele der vorgestellten Internetkonzerne scheint aus der Perspektive des Gesundheitsmarktes die Automobilindustrie zunehmend von Interesse, da das Auto als „third place“ neben der Freizeit und dem Beruf gesehen wird und die Internetkonzerne über die Zeit des Menschen im Auto bislang nur wenig wissen. Und Gesundheit betrifft alle.

\section{Was können Autos in puncto Gesundheit leisten?}

Vergleicht man Medizin mit der Automobilproduktion, so kann potenziell ein höherer Grad der Digitalisierung erreicht werden. Letztendlich besteht die Arbeit des Arztes z.B. zu großen Teilen aus dem Sammeln und Verteilen von Informationen. Autos der Zukunft werden Vitaldaten messen und damit gerade in der Diagnostik eine wesentliche Rolle spielen. Auch ist denkbar, dass ein Arzt während der Autofahrt dazu 
geschaltet wird. Im Notfall kann das Auto bereits jetzt einen Notruf absetzen oder uns (in Zukunft) gleich in die nächste Notaufnahme fahren. Gerade in ländlichen Regionen, in denen es manchmal zu lange dauert, bis der nächste Rettungswagen eintrifft, kann dies Leben retten. Die Arztpraxis wird somit punktuell zu einem rollenden Wartezimmer. Depressionen können durch das Lenkverhalten erkannt und ein Herzinfarkt unmittelbar bei seiner Entstehung diagnostiziert werden. Auch ist in Zukunft denkbar, dass der Blutzuckerspiegel kontinuierlich durch Sensoren überwacht wird. Erste Geräte gibt es bereits. Das Auto der Zukunft erkennt, wann wir Stress haben und kann dann hilfreiche Tipps (beispielsweise Atemübungen) geben, um diesen zu reduzieren. Biofeedback nennt man das neudeutsch. Spannend wird der Zugewinn an Information, wenn man unterschiedliche Daten (Biodaten, Sozialdaten und Verhaltensdaten) miteinander verknüpft - Stichwort: Big Data. Die Aussagemöglichkeiten, die sich hieraus ergeben, können, dürften wir derzeit nicht einmal ansatzweise abschätzen können.

\section{Welche Daten können wir heute schon im Auto erfassen?}

- EKG und Blutdruck/Puls

- Hautschweiß und Hautleitwiderstand

- Körpertemperatur und Temperatureinstellungen

- Fahrerdaten vom Sitz (anthropometrische Daten wie Sitz-, Lenkrad und Spiegeleinstellungen, Gewicht, Bewegung)

- Lenkverhalten (nicht nur zur Müdigkeitserkennung)

- Augenbewegungen

- Stimme

- Geräusche (Atemgeräusche, Husten)

- Geruch

- Nano-Partikel

- Kräfte (Lenkkräfte und Pedalkräfte)

- Reaktionszeiten

- Kommunikationsverhalten u.v.m.

Es ist erkennbar, dass sehr viele Hersteller sich hier auf den Weg gemacht haben. Hierzu zählen Audi, Volkswagen, Daimler, Hyundai, Opel und viele mehr.

\section{Beispiel Audi}

Audi setzt sich für das „emphatische Auto“ ein. Unter dem Motto „my Audi cares for me" soll Audi FitDriver im Fitness- und Gesundheits-Monitoring eingesetzt werden (s. Abb. 2). Die Vision von Audi ist ein Fahrer, der am Ziel entspannter aus dem Auto steigt, als er eingestiegen ist. Dabei stellt das Projekt das Wohlbefinden und die Gesundheit des Fahrers in den Mittelpunkt, indem Vitaldaten erkannt werden und das Fahrzeug sich vitalisierend oder auch schützend auf den Fahrer einstellt. In Zukunft soll das Auto einen Nothalt durchführen und per eCall-System einen Notruf absetzen. 


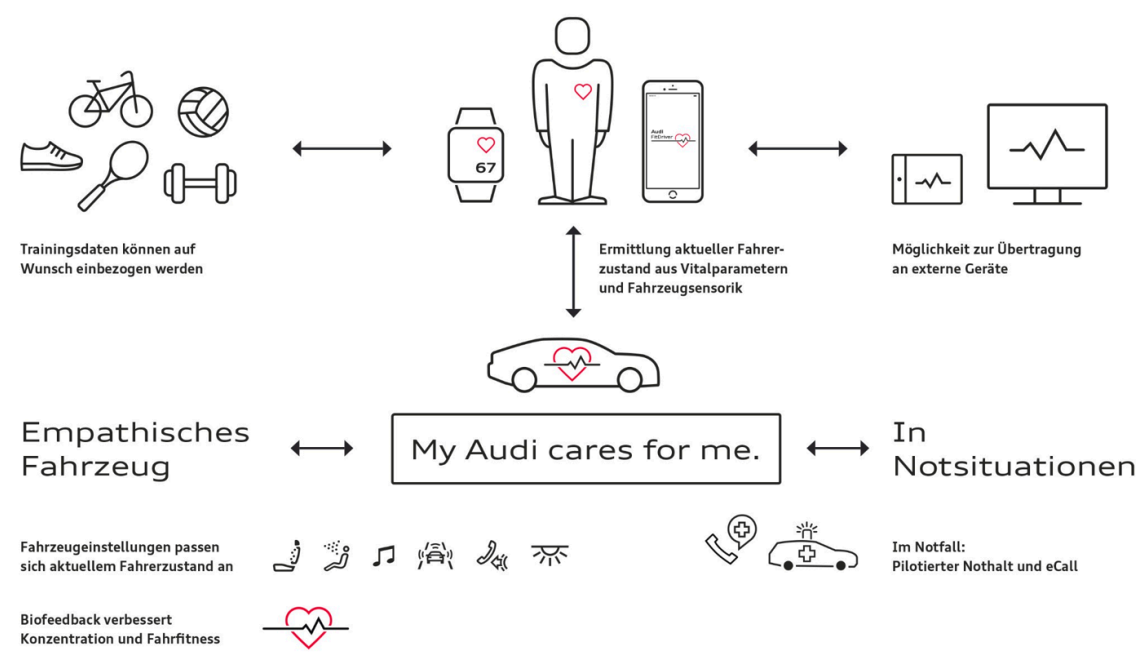

Abb. 2 Audi FitDriver - Funktionen (mit freundlicher Genehmigung der AUDI AG)

\section{Für wen sind die Gesundheitsdaten interessant?}

Interessenten für die Nutzerdaten dürfte es viele geben. Die Autohersteller/Mobilitätsanbieter selbst. Für die Kranken(-versicherungen) sind Risikoprofile interessant. Die Anbieter von Gesundheitsleistungen sehen gerade in der Diagnostik und Prävention Potenziale und später ggfs . auch in der Therapie: Ärzte können in Zukunft ggfs. das Arztgespräch im Auto führen. Für Rettungsdienste sind die Gesundheitsdaten ebenso interessant, und das Krankenhaus wird in Zukunft vielleicht durch selbstfahrende Autos einen neuen Zuweiser für sich entdecken. Dies führt allerdings dazu, dass die Kliniken ihre Notaufnahmen in Zukunft umbauen müssten, da eine Vielzahl von ,privaten Rettungswagen“ logistisch abgefertigt werden müssten. Gesundheitsdaten sind ebenso eine Währung für Amazon, Google, AliBaba \& Co, da Gesundheit das Konsumentenverhalten verändert und bislang ist den IT-Riesen nicht bekannt, was die Kunden während ihrer Autofahrt so tun. Auch die Politik hat Interesse an den Daten, um mehr über den Autofahrer und deren gesundheitlichen Fertigkeiten zum Autofahren zu erfahren. Dies gilt ebenso für die Forschung, die die Epidemiologie, Demografie und anthropometrischen Untersuchungen im Blickfeld hat.

Es stellt sich auch die Frage, ob die Autokunden das überhaupt wollen - ständiges Monitoring/Überwachung ohne direkte Veranlassung. Letztendlich wird sich auch hier die Frage nach dem Gegenwert für den Kunden stellen. Aber Google, Amazon und Facebook machen es ja vor: wenn der Nutzer einen - in seiner Sicht adäquaten Gegenwert für seine Daten bekommt, dann ist er auch bereit, Daten gegen Dienstleistung zu tauschen. Oder führt die Überwachung dazu, dass man Angst hat in sein Auto zu steigen? Der Konsument wird mit den Füßen abstimmen, ob er diese Services haben möchte auf dem „Highway to Health“ oder die Gadgets dann doch eher der Theorie überlässt. 


\section{Ausblick}

Die digitale Transformation wird sowohl die Automobilindustrie als auch das Gesundheitswesen deutlich verändern. Beide Branchen werden eine deutliche Schnittstelle aufweisen, die einen beidseitigen Nutzen hat. Schnittstellemanager in den Automobilkonzernen werden benötigt, die beide Sprachen sprechen. Die Automobilkonzerne werden den Kunden auch aufgrund neuer Konkurrenzen auf dem Markt einen Mehrwert bieten müssen. Das Gesundheitswesen wiederum wird davon profitieren, da die Mobilität für die Versicherten und Patienten in Zukunft eine noch größere Rolle einnehmen wird und dies ebenso einen interessanten Markt darstellen wird. Die Betriebskrankenkassen (insbesondere die Kassen der Automobilkonzerne: Audi BKK, Daimler BKK, BMW BKK) bilden heute schon eine wichtige Schnittstelle zwischen den Automobilkonzernen als Trägerunternehmen und dem Gesundheitsmarkt. Hier sind ebenso wichtige Impulse zu erwarten. Auch das Produkt der Gesundheitswirtschaft wird sich wandeln. Werden bisher die Bereiche kurative Medizin (hauptsächlich 1. Gesundheitsmarkt) und Prävention (oft 2. Gesundheitsmarkt) angeboten, so wird sich die Nachfrage nach „Optimierung“ als umfassendere Kategorie entwickeln. Und das auch während der Autofahrt. Die Potenziale von mobile Health sind in ihrer Entwicklungspotenz noch nicht ausgeschöpft. Derzeit befinden wir uns in einer Zeit- und Branchenschmelze.

Die Digitalisierung der Gesundheit wird (auch) für die Automobilindustrie auf mindestens drei Ebenen relevante Veränderungen ergeben:

1. Mitarbeiterebene: Eine veränderte Gesundheitslandschaft hat direkte Auswirkungen auf die Gesundheitsversorgung und das Gesundheitsverhalten der Mitarbeiter und darüber Auswirkungen für deren Produktivität.

2. Kundenebene: Geht es heute meist darum, das Produkt Auto möglichst wenig gesundheitsschädlich zu machen (siehe auch Feinstaubdebatte) so wird in Zukunft die Einstellung der Kunden zur Mobilität auch davon bestimmt werden, ob diese mit dem Aspekt der Gesundheit einher geht.

3. Geschäftsmodelle: Die Digitalisierung schafft neue Angebote im Health-Bereich und somit eine veränderte/wachsende Nachfrage für Gesundheitsdaten.

Der Beitrag enthält die persönlichen Meinungen der Autoren, nicht der Institutionen.

\section{Literatur}

Lummer S (2017) Automotive Health - Rolling Phones. In: BKK Magazin, 1/2017, S. 18-39 